\title{
Enmiendas orgánicas en la inmovilización de cadmio en suelos agrícolas contaminados: una revisión
}

\author{
Jhon N. Huaraca-Fernandez'1 , Lourdes Pérez-Sosa1, Leonor S. Bustinza-Cabala1 y Noé B. Pampa-Quispe ${ }^{1,2 \star}$ \\ (1) Escuela Profesional de Ingeniería Ambiental, Facultad de Ingeniería y Arquitectura, Universidad Peruana Unión, \\ Carretera Central Km 19.5, Ñaña, Lima-Perú (correo-e: jhonhuaraca@upeu.edu.pe, lourdesperez@upeu.edu.pe, \\ leonorb@upeu.edu.pe) \\ (2) Departamento Académico de Ingeniería, Universidad Nacional de Barranca, Av. Luzuriaga Mz J, La Florida, \\ Lima-Perú (correo-e: npampa@unab.edu.pe) \\ * Autor a quien debe ser dirigida la correspondencia.
}

Recibido Dic. 30, 2019; Aceptado Mar. 2, 2020; Versión final Abr. 26, 2020, Publicado Ago. 2020

\begin{abstract}
Resumen
El presente artículo es una revisión sobre el uso de las enmiendas orgánicas en la inmovilidad del cadmio en suelos agrícolas. El cadmio (Cd), se ha convertido en un problema global desde la industrialización y la implementación de prácticas agrícolas intensivas. En la actualidad, sigue siendo un problema ambiental omnipresente ya que es un metal altamente tóxico que persiste en el suelo por largos periodos debido a su mínima pérdida microbiana y química. Actualmente, existe conciencia pública sobre las implicaciones de los suelos agrícolas contaminados con cadmio y por ello ha aumentado el interés en desarrollar y/o aplicar técnicas rentables, amigables y respetuosas con el ambiente como la inmovilización in situ utilizando enmiendas orgánicas. Se concluye que las enmiendas orgánicas tienen el valor potencial para reducir la captación de Cd por las plantas y mejorar la inmovilización por procesos de adsorción, intercambio iónico, complejación y precipitación.
\end{abstract}

Palabras clave: biochar; biodisponibilidad; compost; estiércol; suelo; vermicompost

\section{Organic amendments in the immobilization of cadmium in contaminated agricultural soils: a review}

\begin{abstract}
This article is a review of the use of organic amendments in the immobility of cadmium in contaminated agricultural soils. Cadmium (Cd) has become a global problem since industrialization began and since the implementation of intensive agricultural practices. Cadmium remains as an omnipresent environmental problem since it is a highly toxic metal that persists in the soil for long periods due to its minimal microbial and chemical loss. Currently, there is public awareness about the implications of agricultural soils contaminated with cadmium. Therefore, there is increased interest in developing and applying cost-effective, friendly, and environmentally friendly techniques such as in situ immobilization using organic amendments. It is concluded that organic amendments have the potential value to reduce Cd uptake by plants and improve immobilization by adsorption, ion exchange, complexation and precipitation.
\end{abstract}

Keywords: biochar; bioavailability; compost; manure; soil; vermicompost 


\section{INTRODUCCIÓN}

El cadmio (Cd) es un metal pesado sin funciones biológicas esenciales, que causa gran preocupación en el medio ambiente debido a su toxicidad para los animales y los seres humanos; las concentraciones de Cd pueden acumularse en plantas que no son tóxicas para ellas, pero son tóxicas para los animales y las personas que los consumen. Entre todos los metales pesados tóxicos, el Cd es el más móvil y biodisponible (Gramlich et al, 2017; Gramlich et al, 2018; Lewis et al., 2018). En los seres humanos, la exposición al Cd se ha relacionado con la disfunción renal, la osteoporosis y varios tipos de cáncer (Åkesson et al., 2014). La presencia de $\mathrm{Cd}$ en el suelo se produce tanto de fuentes naturales como antropogénicas (Pan et al., 2016; Khan et al., 2017).

En la agricultura, la principal fuente de este metal es la aplicación de fertilizantes que contienen fosfatos, estos hacen que el $\mathrm{Cd}$ se acumule en suelos agrícolas y por lo tanto aumente el riesgo de su transferencia a través de la cadena alimentaria (Roberts, 2014; Salmanzadeh et al., 2017). Hoy en día, se necesitan urgentemente técnicas factibles para remediar los suelos contaminados con $\mathrm{Cd}$ y con ello; garantizar la inocuidad de los alimentos y proteger la salud pública (Guo et al., 2018a). Se han investigado varias técnicas de remediación de suelos en experimentos de campo y en ambientes controlados, como el lavado de suelos, la fitorremediación, la solidificación, la estabilización, la excavación y electrorremediación; estas técnicas de remediación emplean mecanismos de contención, extracción/eliminación e inmovilización para reducir los efectos de contaminación a través de procesos físicos, químicos, biológicos, eléctricos y térmicos (Tajudin et al., 2016; Liu et al., 2018a). Sin embargo, la mayoría de estas técnicas pueden producir efectos secundarios al aumentar la movilidad de los metales y cambiar las propiedades físico-químicas del suelo.

Para los suelos agrícolas contaminados con cadmio, la técnica de inmovilización química in situ denominada también como estabilización in situ implica la aplicación de enmiendas para inmovilizar metales a través de diversas reacciones biológicas y químicas, representa una solución más realista, rentable, simple, con rápida implementación y sostenibilidad ambiental (Hu et al., 2016; Sun et al., 2016; Khan et al., 2017), dado que de forma rutinaria a nivel mundial cada día se generan grandes volúmenes de desechos orgánicos de la agricultura, de las industrias de ganado y aves de corral, estos materiales pueden proporcionar grandes cantidades para tratar extensas áreas de tierras agrícolas (Cruz-Paredes et al., 2017; Khan et al., 2017). Esta técnica consiste en atrapar o inmovilizar el Cd en el suelo mediante la introducción de agentes estabilizadores como las enmiendas, para solidificar el suelo o convertir las fracciones de contaminantes móviles; es decir, formas solubles e intercambiables en precipitados y/o restos fuertemente absorbidos (Tajudin et al., 2016).

La mayoría de las enmiendas orgánicas disminuyen el Cd disponible para las plantas, principalmente, a través de la adsorción, sorción, precipitación y los procesos de formación de complejos (Shaheen et al., 2014). Asimismo, las enmiendas orgánicas forman un recubrimiento sobre una materia particulada especialmente en las capas superficiales y sub superficiales del suelo y podrían actuar como aglomerantes metálicos. Estas enmiendas se caracterizan por la presencia de lignina, celulosa, taninos y carbonatos que aumentan la capacidad natural del suelo para retener metales pesados (Sebastian y Prasad, 2013).

La inmovilización química no elimina ni extrae contaminantes del suelo, se basa en la movilidad/solubilidad de los metales pesados y sus concentraciones en el agua de los poros del suelo, disminuyendo su concentración drásticamente, lo que minimiza su posible transporte a plantas, microorganismos y aguas (Tajudin et al., 2016). El presente artículo aborda la revisión sobre el empleo de las enmiendas orgánicas en la inmovilidad del cadmio en suelos agrícolas contaminados.

\section{EL ELEMENTO CADMIO Y BIODISPONIBILIDAD}

El cadmio es un metal pesado tóxico sin funciones biológicas esenciales para las plantas, animales y humanos, se encuentra principalmente como un catión divalente, que forma complejos con otros aniones. Está clasificado en la $7^{\underline{a}}$ posición entre 20 toxinas, debido a su alta solubilidad y naturaleza tóxica (Gramlich et al., 2018; Hamid et al., 2018). Se emplea ampliamente en baterías, pinturas, pantallas de televisión, paneles solares, láseres, cosméticos y en los fertilizantes. Comparado con otros elementos pesados, se absorbe fácilmente por las raíces y se transporta hacia los brotes de las plantas (Yang et al., 2017), y de ésta se incorpora a la cadena alimenticia (Guo et al., 2018b). La toxicidad del cadmio en las plantas causa retraso en el crecimiento, clorosis, enrojecimiento de las puntas de las raíces y conduce a su muerte (Guo et al., 2008). Los cultivos más tolerantes son: girasol (Helianthus annuus), pak choi (Brassica chinensis L.), Mostaza de la India (Brassica juncea), Noccaea caerulescens (Thlaspi caerulescnes), Arabidopsis thaliana, Arabis paniculata, Arabis gemmifera, N. goesingensis (T. goesingense) y N. praecox (T. praecox). 
El cadmio se libera al medio ambiente en cantidades variables por actividades naturales y antropogénicas. Los estudios realizados muestran que la contribución total de las fuentes naturales a la contaminación de Cd en el suelo representa el 10\% en comparación a las fuentes antropogénicas (Cloquet et al., 2006). Entre las principales fuentes naturales de $\mathrm{Cd}$ a la atmósfera se encuentran las rocas progenitoras, las erupciones volcánicas, los incendios forestales, el polvo arrastrado por el viento y la pulverización marina (Liu et al., 2013). Las fuentes antropogénicas liberan al medio ambiente más del $90 \%$ de Cd y las fuentes principales son el uso de fertilizantes fosfatados, detergentes, combustión de combustibles fósiles, trabajos metalúrgicos, desechos de la industria del cemento, lodos de depuración, desechos municipales e industriales, minería, fundición y procesamiento de minerales (Cloquet et al., 2006).

El término biodisponibilidad se define como "la parte de la concentración total de una sustancia química disponible para las células receptoras (plantas, microorganismos, etc.)". Por ello, el Cd biodisponible es la fracción del $\mathrm{Cd}$ total en el agua intersticial y las partículas del suelo que está fácilmente disponible para los organismos receptores (Khan et al., 2017). La parte de la concentración de Cd del suelo disponible para las plantas se denomina $\mathrm{Cd}$ biodisponible. Esta concentración biodisponible es la principal preocupación por su absorción y acumulación en las plantas. Por ello, en cualquier estudio, se deben medir tanto la concentración total como la concentración biodisponible. Los principales factores que influyen en la biodisponibilidad de $\mathrm{Cd}$ en el suelo son: el potencial de hidrógeno $(\mathrm{pH})$ del suelo, la presencia de materia orgánica y la capacidad de intercambio iónico (tabla 2). Estos factores influyen en la solubilidad de los compuestos de $\mathrm{Cd}$ y la liberación de $\mathrm{Cd}$ en la solución del suelo o afectan la capacidad de las plantas para absorber el Cd del suelo (Sarwar et al., 2010).

El pH es uno de los factores más importantes, que controla la absorción de cadmio, a medida que el pH disminuye, aumenta la absorción del Cd por las plantas. Es decir, que existe una relación lineal indirecta. Al incrementar el $\mathrm{pH}$ en el suelo, el cadmio es removido y adsorbido por los coloides del suelo, restringiendo su movilidad y biodisponibilidad. Del mismo modo, Liu et al. (2019) como Shen et al. (2019) declararon que las enmiendas orgánicas, especialmente, las dosis altas, aumentan el $\mathrm{pH}$ del suelo y el contenido de materia orgánica, y en consecuencia disminuyen la biodisponibilidad de Cd del suelo.

La materia orgánica es un componente reactivo importante en suelos capaces de retener cationes metálicos (He et al., 2015). La aplicación de materia orgánica a un suelo contaminado puede reducir la capacidad de extracción y la biodisponibilidad de $\mathrm{Cd}, \mathrm{Zn}$ y $\mathrm{Pb}$ (Houben et al., 2012). En estudios realizados por $\mathrm{He}$ et al. (2015) se muestra que la presencia de $5 \%$ y $10 \%$ de biochar disminuye las concentraciones extraíbles de $\mathrm{Cd}, \mathrm{Zn}$ y $\mathrm{Pb}$ durante los siguientes 56 días. Hao et al. (2012) informaron que el estiércol de cerdo disminuyen significativamente las concentraciones de $\mathrm{Cd}$ y $\mathrm{Zn}$ en el girasol. Los residuos orgánicos como estiércol de granja, estiércol de aves de corral, estiércol de cerdo, compost, biosólidos, lodo de alcantarillado, desechos domésticos, paja y una amplia gama de enmiendas orgánicas combinadas pueden utilizarse como enmiendas del suelo para reducir la disponibilidad de Cd (Sarwar et al., 2010; Hao et al., 2012).

La capacidad de intercambio catiónico $(\mathrm{CIC})$ es un fenómeno basado en la presencia de cargas en las arcillas y demás partículas coloidales de los suelos. Por medio de estas cargas, los iones que son liberados de minerales que han sido sometidos a procesos de meteorización o provenientes de compuestos orgánicos en descomposición, del agua de lluvia o de riego y de los fertilizantes, pueden ser absorbidos por las partículas del suelo y bajo esta condición son retenidos en forma relativamente inmóvil. Asimismo, He et al. (2015) afirman que la capacidad de intercambio catiónico es un factor importante que influye en la movilidad de $\mathrm{Cd}$ en el suelo y que este elemento está fuertemente ligado a las superficies de minerales de arcilla, óxidos de $\mathrm{Fe}, \mathrm{Al}$ y humus, disminuyendo así su movilidad en suelos arcillosos.

Los suelos que presentan alta capacidad de intercambio catiónico (ricos en materia orgánica y con elevados contenidos de arcilla), por ejemplo: suelos humíferos y arcillosos, dan como resultado la inmovilización de los metales pesados por quedar estos retenidos por adsorción al complejo coloidal del suelo (Cortes Páez et al., 2016).

\section{ABSORCIÓN DEL CADMIO POR LAS PLANTAS}

Las plantas toman $\mathrm{Cd}$ del agua intersticial del suelo. Si bien la mayor parte del $\mathrm{Cd}$ en los suelos generalmente se asocia con los coloides del suelo $(>98 \%)$, con solo una pequeña fracción del total está disponible para la absorción. Al manipular las cantidades de sitios de unión de cationes disponibles en los suelos, la cantidad de Cd disuelto se puede reducir y disminuye su absorción por las plantas. Esto se puede lograr con el cambio del pH del suelo (encalado) o mediante la introducción de sitios de unión adicionales (Kabata-Pendias y Mukherjee, 2007). 
Las enmiendas orgánicas (aserrín, biochar, turba, compost, etc.) pueden reducir efectivamente la solubilidad del Cd y su absorción por las plantas en suelos altamente contaminados. El Cd en el suelo forma quelatos con materia orgánica reduciendo su fitodisponibilidad (Singh y Prasad, 2014). Khan et al. (2018) investigaron los efectos de diferentes enmiendas orgánicas (biochar de madera dura, bagazo, cáscara de arroz y residuos de mazorca de maíz) sobre la biodisponibilidad de $\mathrm{Cd}$ en suelos contaminados por metales pesados y su posterior bioacumulación en tomate y pepino. Entre las enmiendas utilizadas, el biochar de madera dura fue la más efectiva para reducir la biodisponibilidad de $\mathrm{Cd}$, en donde se observaron disminuciones significativas en la absorción de este elemento por el tomate (24-30\%) y pepino (36-54\%). Se demostró que a mayor tasa de aplicación de enmiendas orgánicas, es más efectiva para mitigar la movilidad de Cd y la bioacumulación en plantas cultivadas en suelos que contienen Cd.

Las investigaciones de Yu et al. (2018); Liu et al. (2019) y Rehman et al. (2019) mostraron que la aplicación de enmiendas al suelo disminuyen los niveles de $\mathrm{Cd}$ en las plantas. Además, señalan que una combinación de enmiendas orgánicas podría ayudar a reducir aún más los niveles de $\mathrm{Cd}$, en comparación con las enmiendas orgánicas individuales. Este progreso de mitigación podría atribuirse a los cambios en la translocación y distribución de Cd entre los tejidos de las plantas y la inhibición de la biodisponibilidad de Cd en el suelo a través de la alteración de las propiedades del suelo $(\mathrm{pH}, \mathrm{ClC}, \mathrm{MO})$. Las enmiendas orgánicas combinadas facilitaron la transformación del $\mathrm{Cd}$ del suelo de la forma biodisponible a la forma de $\mathrm{Cd}$ inmovilizado, y por lo tanto disminuyeron los niveles de $\mathrm{Cd}$ en las plantas, esto se debe a que las enmiendas orgánicas contienen ácidos húmicos (AH). Debido a su carácter anfifílico, los $\mathrm{AH}$ forman estructuras parecidas a micelas en condiciones neutras a ácidas, que son útiles en agricultura, para la remediación de suelos contaminados con metales pesados. Las AH tienen composiciones indefinidas que varían según el origen, el proceso de obtención y los grupos funcionales presentes en sus estructuras, como quinonas, fenoles y ácidos carboxílicos (De Melo et al., 2016).

Los estudios realizados por Nieto-Velázquez et al. (2011) muestran claramente la existencia de interacciones entre los ácidos húmicos y los metales pesados. Estas interacciones pueden ser de utilidad para la inmovilización de metales pesados en aguas y suelos como estrategia de remediación de la contaminación. Las enmiendas orgánicas contienen sustancias húmicas, estas pueden reducir la movilidad y la bioaccesibilidad del Cd en el suelo y, por lo tanto, inhibir su absorción por las plantas (Yu et al., 2018).

Las tablas 1 y 2 muestran respectivamente, los factores edáficos y del cultivo que determinan la absorción de cadmio en las plantas y la relación que genera la bioacumulación de cadmio en los granos y frutos de las plantas, respectivamente.

Tabla 1: Factores edáficos y del cultivo que determinan la absorción de Cadmio en las plantas (Adaptado MINAGRI, 2018).

\begin{tabular}{|l|l|}
\hline Factores & Efecto en la absorción de Cadmio por las plantas. \\
\hline Factores edáficos & La absorción se incrementa cuando disminuye el pH (suelos ácidos). \\
\hline $\mathrm{pH}$ & $\begin{array}{l}\text { La absorción se incrementa con la concentración de Cd, relación } \\
\text { directa. }\end{array}$ \\
\hline Cantidad de cadmio & $\begin{array}{l}\text { Puede incrementar (deficiencia de micronutrientes) o decrecer } \\
\text { (concentración adecuada de micronutrientes) la absorción. }\end{array}$ \\
\hline Micronutrientes & $\begin{array}{l}\text { Puede incrementar (deficiencia de macronutrientes) o decrecer } \\
\text { (concentración adecuada de macronutrientes) la absorción. }\end{array}$ \\
\hline Macronutrientes & Alta temperatura incrementa la absorción. \\
\hline Temperatura & \multicolumn{2}{|l|}{} \\
\hline Factores del cultivo & $\begin{array}{l}\text { Las hortalizas absorben más que las raíces, las raíces absorben más } \\
\text { que los cereales, y los cereales absorben más que los frutos. }\end{array}$ \\
\hline Especies y cultivares & Hoja > grano > fruto y raíces comestible. \\
\hline Tejido de la planta & Hojas viejas > hojas jóvenes. \\
\hline Edad de la hoja &
\end{tabular}


Tabla 2: Relación de problemas que genera la bioacumulación de cadmio en los granos y frutos de las plantas (Adaptado MINAGRI, 2018)

\begin{tabular}{|c|c|}
\hline $\begin{array}{l}\text { Problemas que generan } \\
\text { la bioacumulación de } \\
\text { Cadmio en las plantas. }\end{array}$ & Medidas de mitigación propuestas. \\
\hline $\begin{array}{l}\text { Suelos de baja fertilidad } \\
\text { natural. }\end{array}$ & Fertilizar el suelo con adecuado contenido de nutrientes. \\
\hline $\begin{array}{l}\text { Bajo contenido de } \\
\text { materia orgánica en el } \\
\text { suelo. }\end{array}$ & Incrementar la materia orgánica (> 4\%MOS). \\
\hline $\begin{array}{l}\text { Baja concentración de } \\
\text { Zn y Mn. }\end{array}$ & Incorporación de cantidades adecuadas de zinc y manganeso. \\
\hline Suelos arenosos. & $\begin{array}{l}\text { Evitar sembrar en suelos arenosos, de preferencia utilizar suelos } \\
\text { francos a arcillosos. }\end{array}$ \\
\hline $\begin{array}{l}\text { Aguas salinas }(2 \mathrm{mS} / \mathrm{cm}) \\
\text { con altos contenidos de } \\
\text { cloruros. }\end{array}$ & Tratar el agua de riego para bajar su salinidad y disminuir los cloruros. \\
\hline $\begin{array}{l}\text { Suelos fuertemente } \\
\text { ácidos. }\end{array}$ & $\begin{array}{l}\text { Encalar los suelos hasta niveles moderadamente neutros. El aumento } \\
\text { en el pH del suelo mejora la disociación de } \mathrm{H}^{+} \text {de los grupos } \\
\text { funcionales tales como hidroxilo, fenólico, carbonilo y carboxilo, } \\
\text { mejorando así la afinidad por los iones de cadmio. }\end{array}$ \\
\hline
\end{tabular}

\section{ENMIENDAS ORGÁNICAS PARA ESTABILIZAR EL CADMIO EN SUELOS}

Una enmienda orgánica es cualquier material orgánico, incluido el compost, los abonos animales, desechos orgánicos, biofertilizantes residuos de cultivos o acondicionadores sintéticos del suelo que se incorporan al suelo o se aplican en la superficie para mejorar el crecimiento de la planta (Hueso-González et al., 2018). Las enmiendas orgánicas tienen el potencial para afectar significativamente el comportamiento de los metales en el suelo alterando su solubilidad, disponibilidad, transporte y distribución espacial. Muchos estudios han demostrado claramente que la aplicación de las enmiendas orgánicas en suelos contaminados con metales tienen el potencial de remediación in situ por inmovilización de metales, reduciendo así la disponibilidad de metal para las plantas (Mohamed et al., 2010; Park et al., 2011). Las enmiendas orgánicas cuentan con una estructura porosa, alto $\mathrm{pH}$, alto $\mathrm{ClC}$ y grupos funcionales que contienen oxígeno, estas características influyen en las propiedades fisicoquímicas del suelo y ayudan en la inmovilización del Cd (Park et al., 2011; Jiang et al., 2012).

Asimismo, las enmiendas orgánicas pueden usarse como sumideros para reducir la biodisponibilidad del metal en suelos y sedimentos contaminados a través de su efecto sobre la adsorción, complejación, intercambio iónico (Tabla 3), precipitación, reducción y volatilización del metal (Park et al., 2011). La retención del metal inducida por la enmienda orgánica se atribuye a un aumento en la carga superficial y la presencia de compuestos de unión de metal (Clark et al., 2007). La inmovilización de Cd mediante enmiendas ofrece un menor transporte y biodisponibilidad en el suelo (Fig. 1).

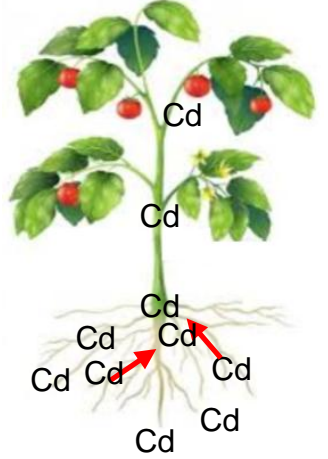

Cadmio biodisponible en el suelo
Papel de las enmiendas orgánicas

Disminuye la biodisponibilidad de $\mathrm{Cd}$. Incrementa el $\mathrm{pH}$ del suelo. Forma complejos estables.

Diferencia

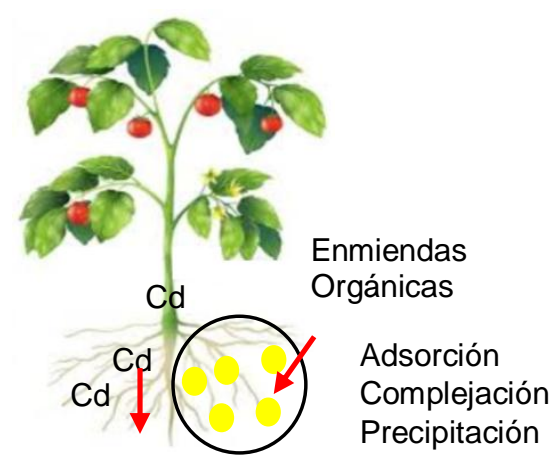

Reducción del Cd biodisponible mediante enmiendas orgánicas

Fig. 1: Reducción del cadmio disponible mediante enmiendas orgánicas. 


\begin{tabular}{|c|c|c|c|c|c|}
\hline 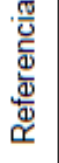 & 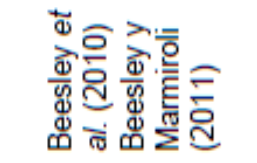 & 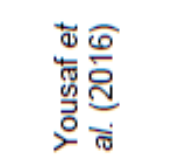 & 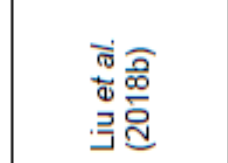 & 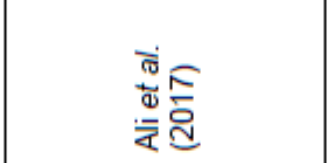 & 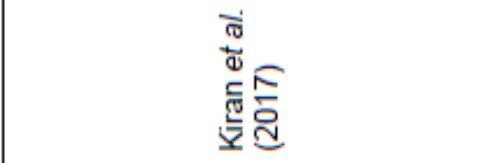 \\
\hline 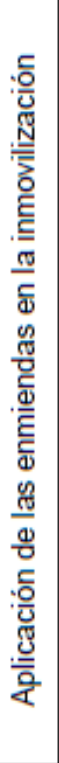 & 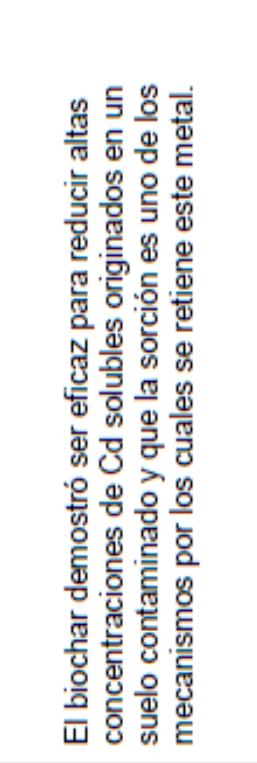 & 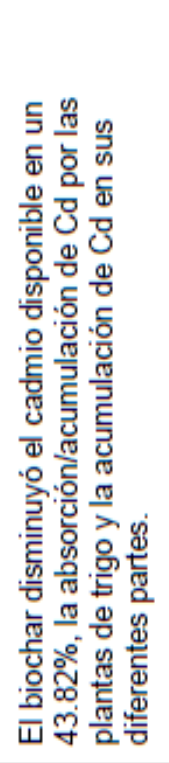 & 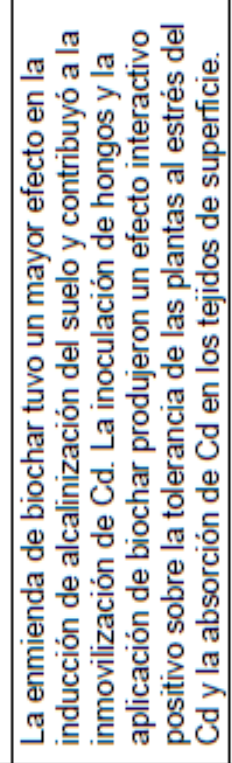 & 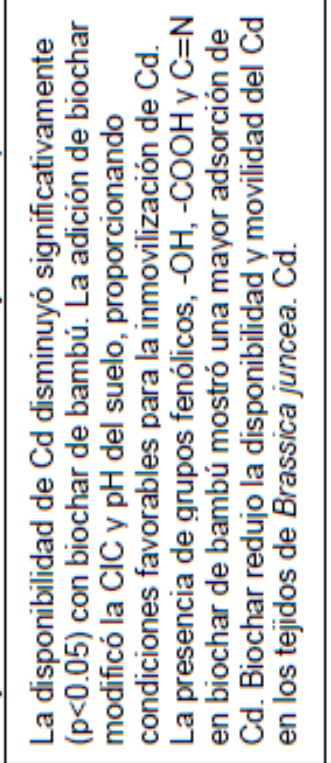 & 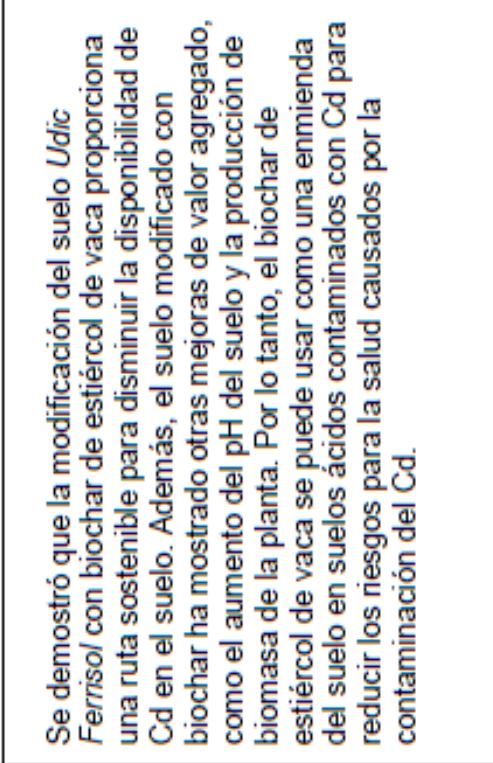 \\
\hline 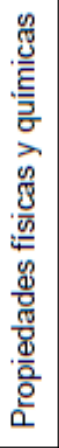 & 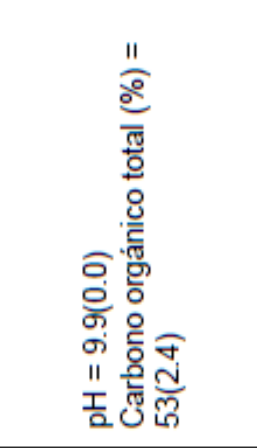 & 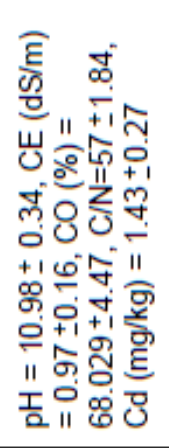 & 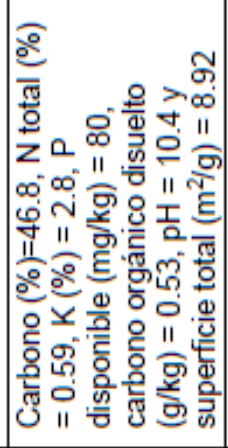 & 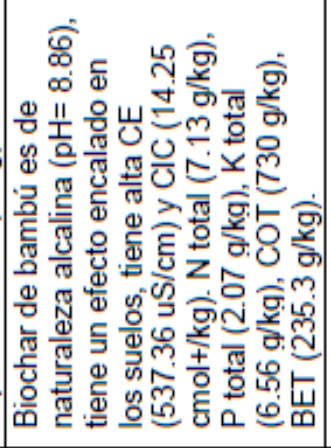 & 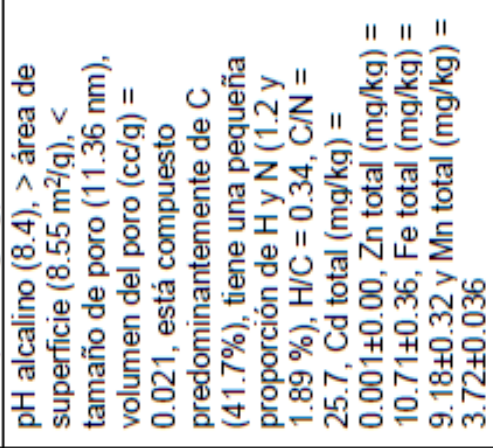 \\
\hline 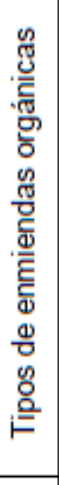 & 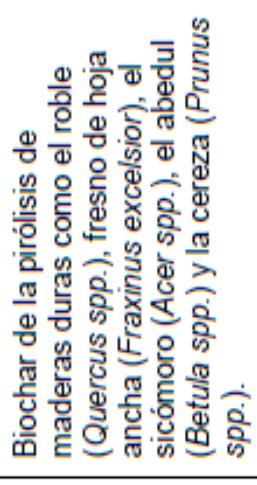 & 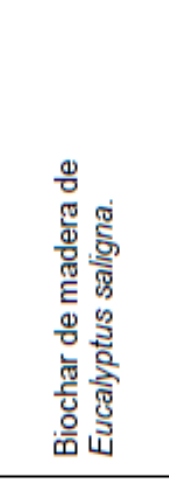 & 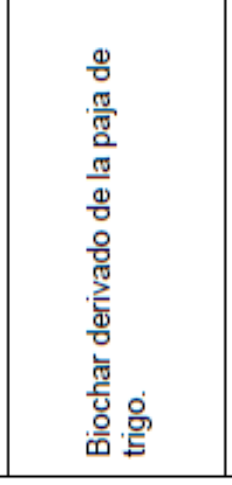 & 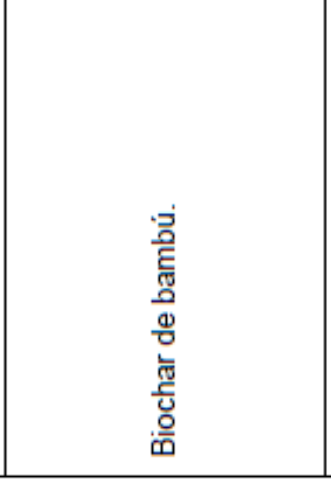 & 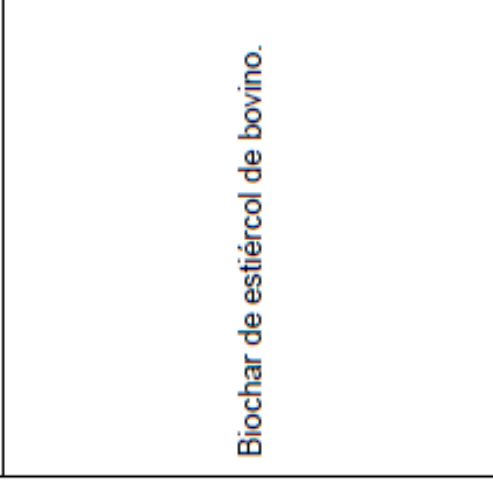 \\
\hline 高 & & 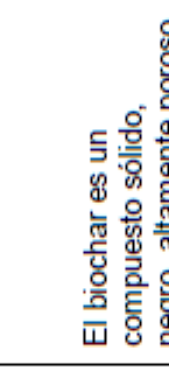 & 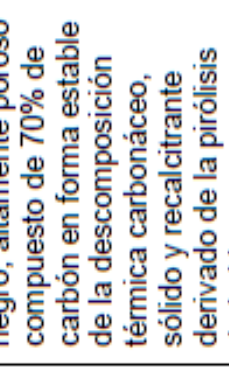 & 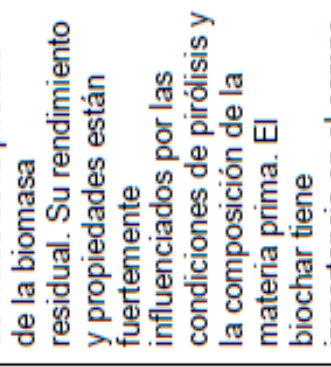 & 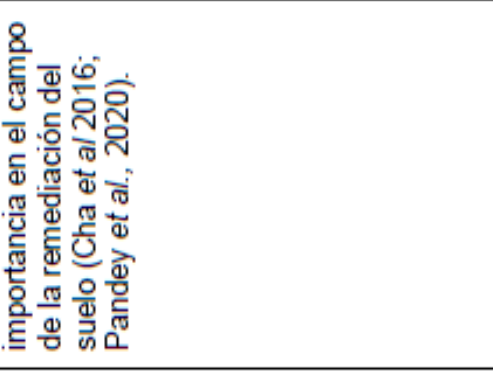 \\
\hline 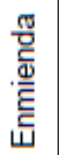 & & & & 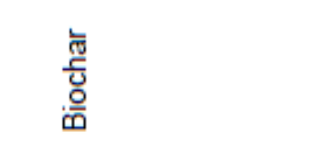 & \\
\hline
\end{tabular}




\begin{tabular}{|c|c|c|c|c|}
\hline 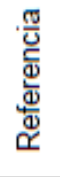 & 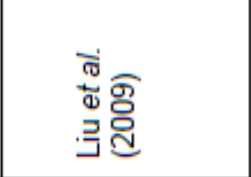 & 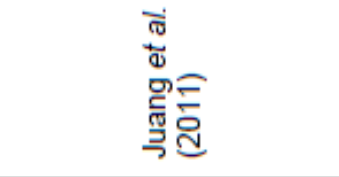 & 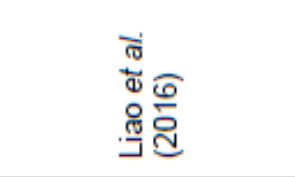 & 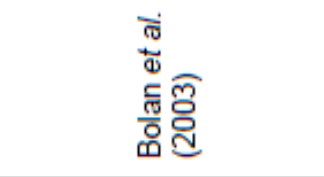 \\
\hline 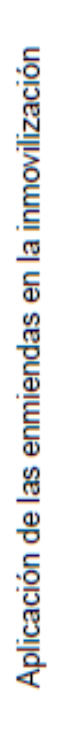 & 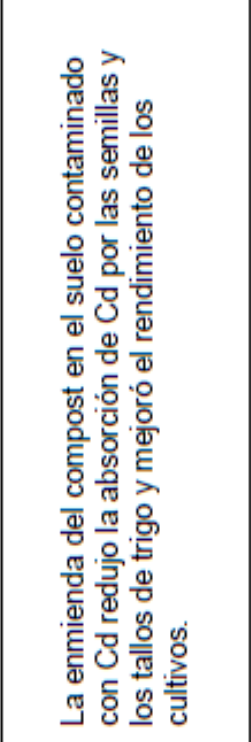 & 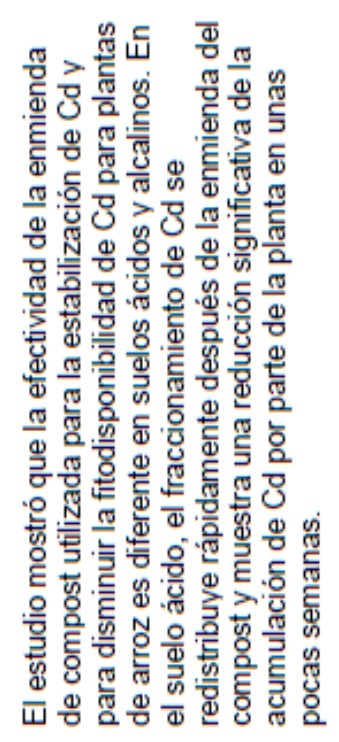 & 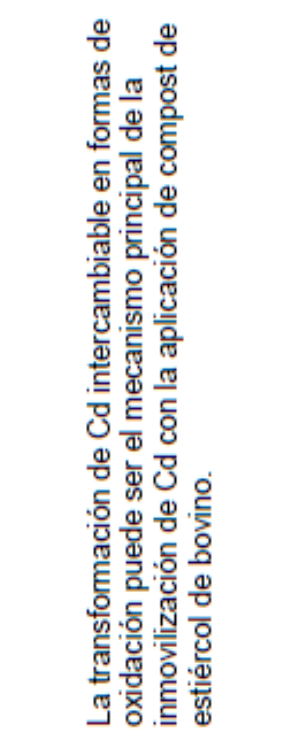 & 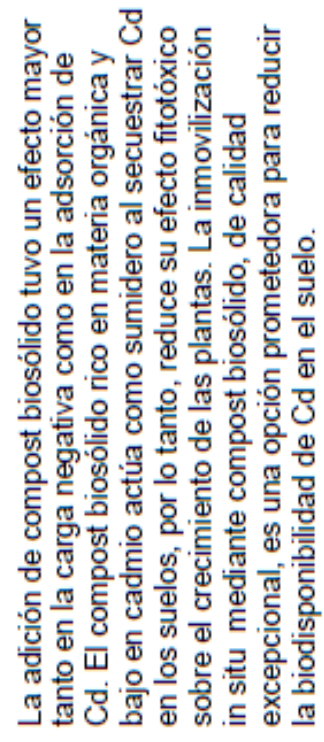 \\
\hline 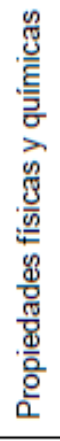 & 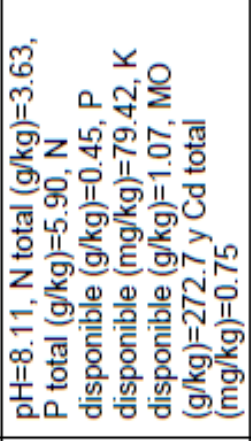 & 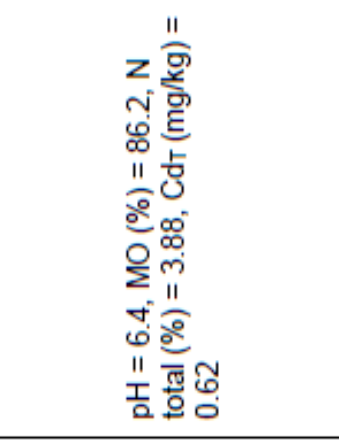 & 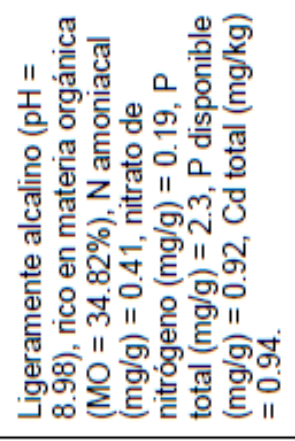 & 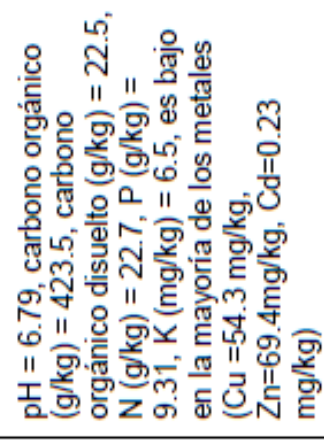 \\
\hline 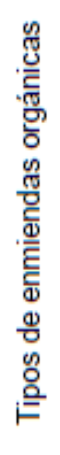 & 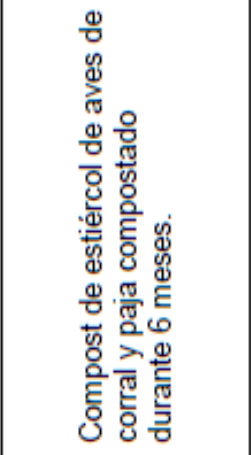 & 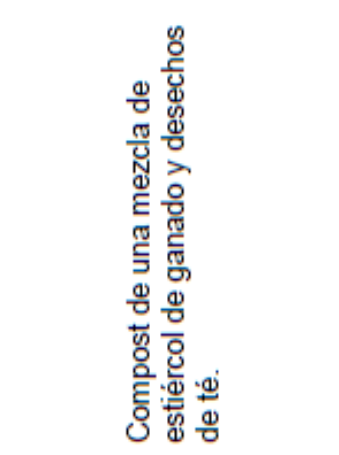 & 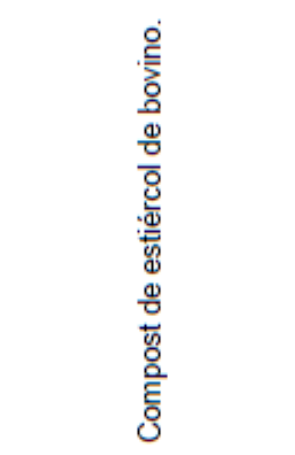 & 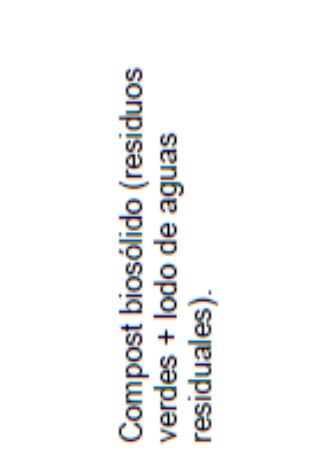 \\
\hline مَّ & \multicolumn{4}{|c|}{ 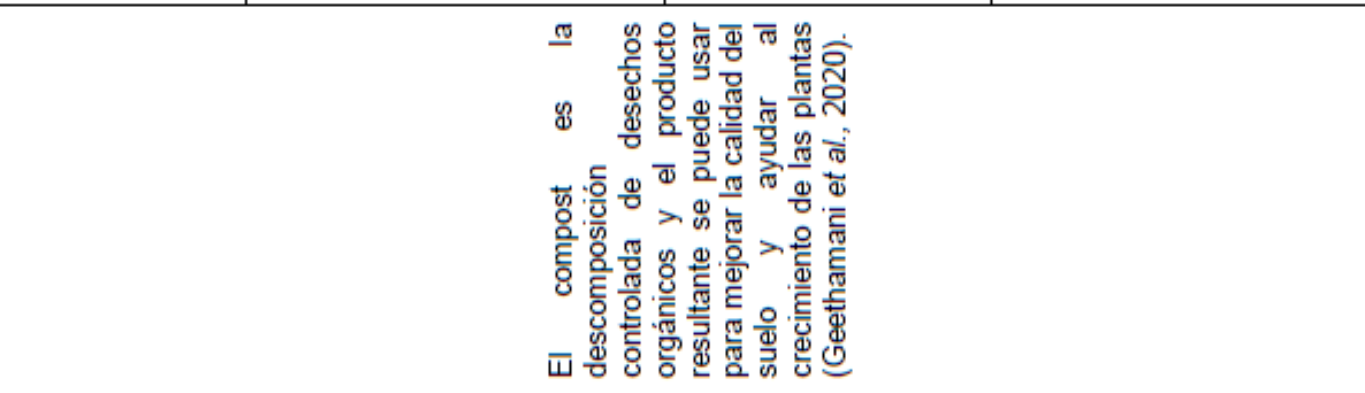 } \\
\hline 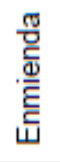 & & & $\begin{array}{l}\text { ". } \\
\text { o. } \\
\text { हे }\end{array}$ & \\
\hline
\end{tabular}




\begin{tabular}{|c|c|c|c|c|c|}
\hline 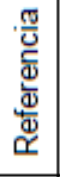 & 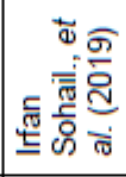 & 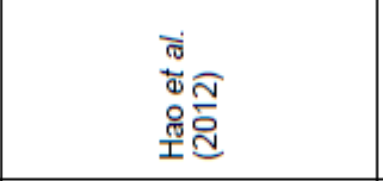 & 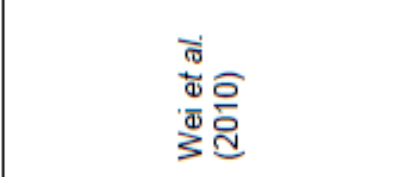 & 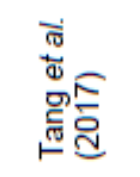 & 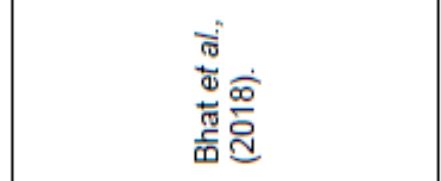 \\
\hline 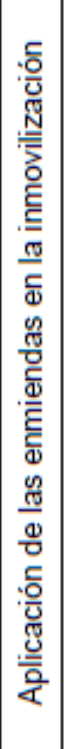 & 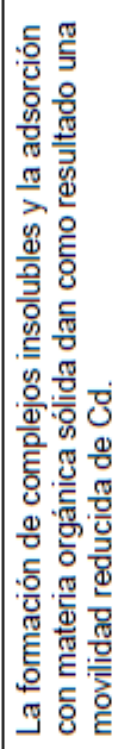 & 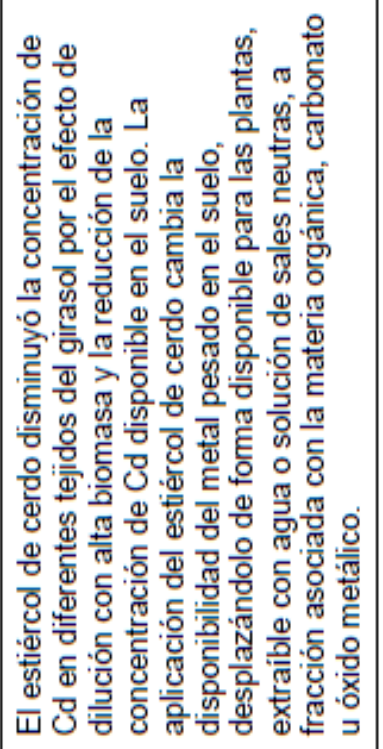 & 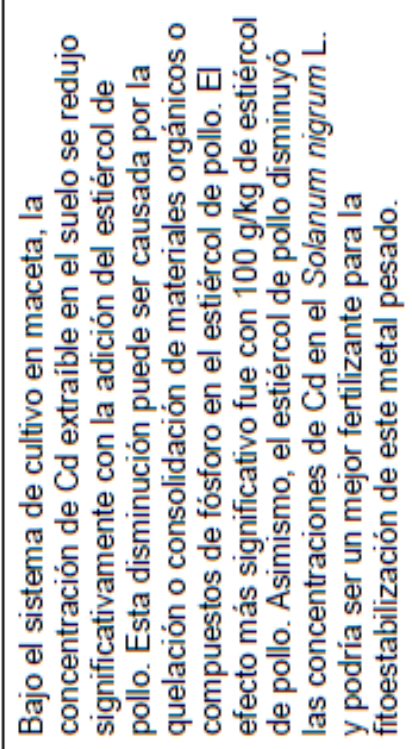 & 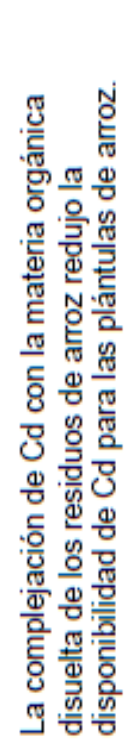 & 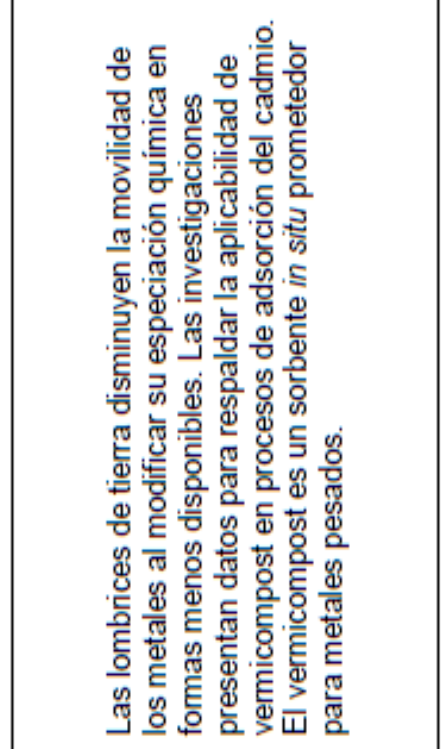 \\
\hline 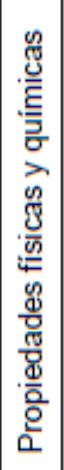 & 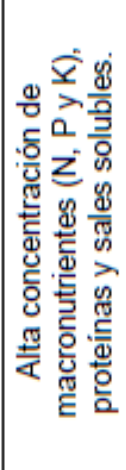 & 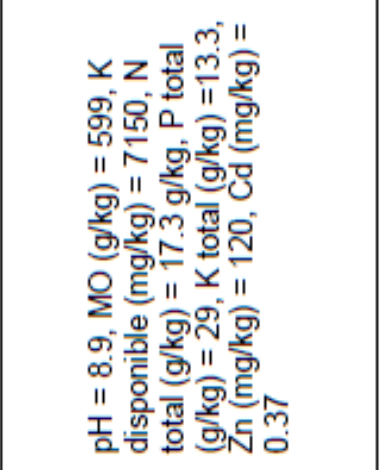 & 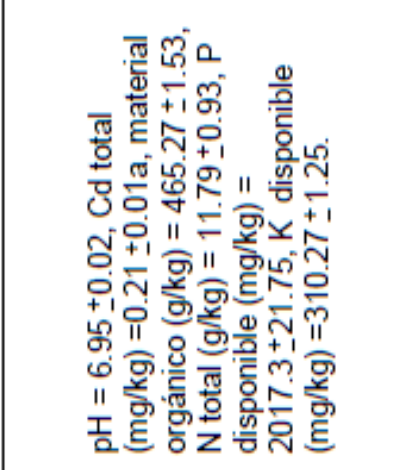 & 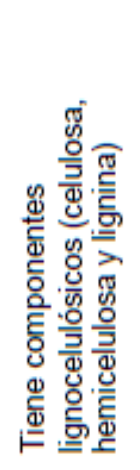 & 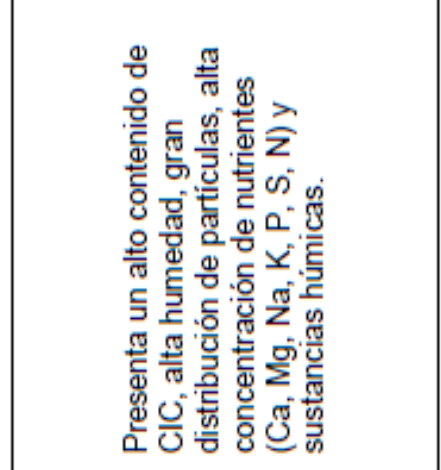 \\
\hline 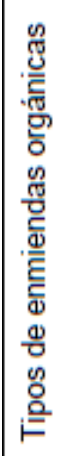 & 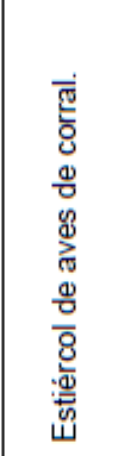 & 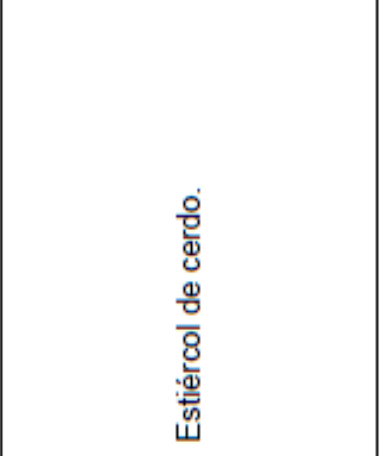 & 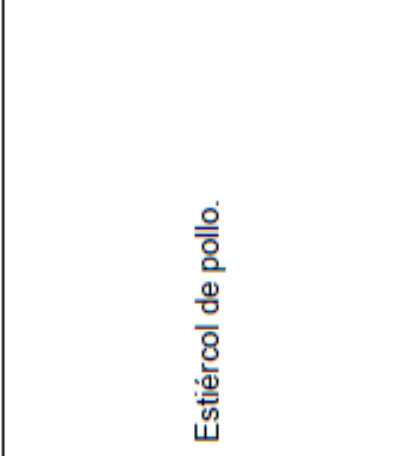 & 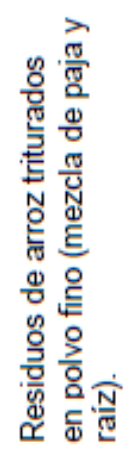 & 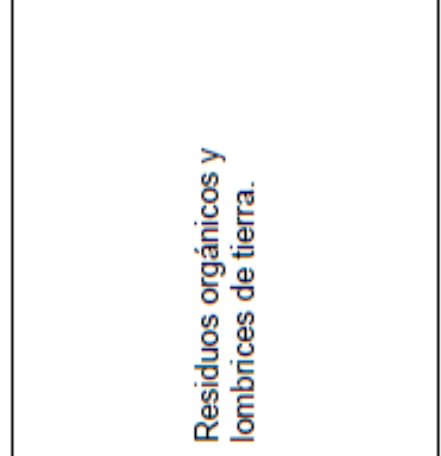 \\
\hline 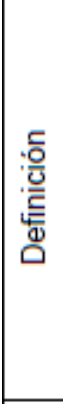 & & 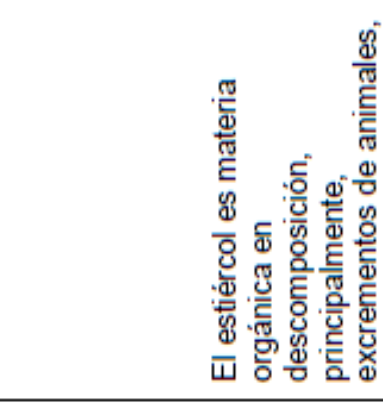 & 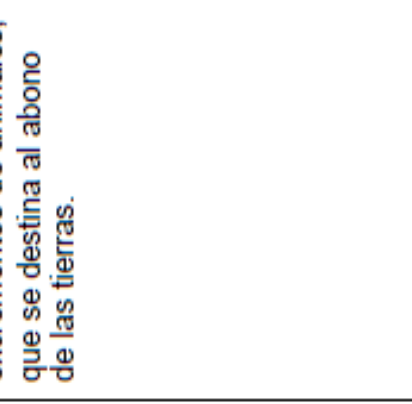 & 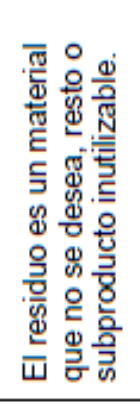 & 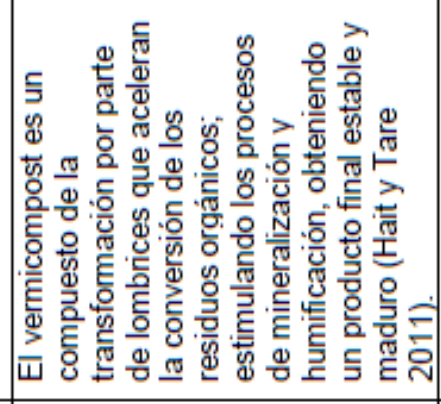 \\
\hline 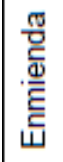 & & 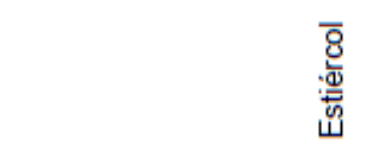 & & 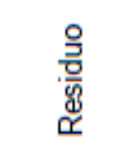 & 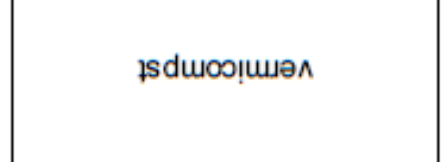 \\
\hline
\end{tabular}


Como se observa en la tabla 3, un número importante de investigaciones ha demostrado que la adición de enmiendas orgánicas a los suelos aumenta la inmovilización de los metales a través de reacciones de adsorción. La retención del metal inducida por la enmienda orgánica se atribuye a un aumento en la carga superficial. Sin embargo, la concentración de $\mathrm{Cd}$ en el suelo no disminuye con el uso de enmiendas orgánicas, pero si reduce su biodisponibilidad. La materia orgánica en el suelo convierte el $\mathrm{Cd}$ soluble/intercambiable en una fracción de enlace orgánico, lo que reduce la absorción (tabla 2).

El aumento en el pH del suelo mejora la disociación de $\mathrm{H}^{+}$de los grupos funcionales tales como hidroxilo, fenólico, carbonilo y carboxilo, mejorando así la afinidad por los iones de cadmio (Bolan et al., 2003). Además, la aplicación de enmiendas orgánicas hace que el Cd sea menos móvil, pero la concentración total sigue siendo la misma y puede aumentar si las enmiendas orgánicas contienen Cd (Chaudri et al., 2007; Liu et al., 2015).

Diferentes agentes estabilizantes (enmiendas) orgánicos se pueden usar para reducir la movilidad de los metales en sitios contaminados (Hamid et al., 2018). Por ejemplo los abonos de estiércol, biosólidos y desechos sólidos municipales con bajo contenido de metal son considerados fuente de nutrientes y también como acondicionador para mejorar las propiedades físicas y la fertilidad de los suelos. A menudo se ha demostrado que la adición de enmiendas orgánicas a los suelos aumenta la inmovilización del metal a través de reacciones de adsorción (Park et al., 2011). La adición de compost biosólido aumenta la carga superficial de los suelos modificados, lo que se atribuye al mayor $\mathrm{pH}$ y la carga superficial del compost biosólido. Investigaciones realizadas demuestran que la concentración de $\mathrm{Cd}$ disponible en el suelo disminuye significativamente debido a la adición de estiércol de pollo (Wei et al., 2010).

Experimentos realizados por Xie et al. (2018) evaluaron 6 enmiendas orgánicas compuestas en la inmovilización del $\mathrm{Cd}$ y reportaron una disminución prominente en el contenido de $\mathrm{Cd}$ y Cu biodisponible. De manera similar, Hamid et al. (2018) evaluaron doce enmiendas (solas y mixtas) para la inmovilización de Cd en el sistema de cultivo de arroz-trigo y encontraron una disminución relativamente mayor en la absorción de la planta por la adición combinada de estiércol.

\section{DISCUSIÓN}

La contaminación de suelos agrícolas con cadmio es un problema global, por lo que la tierra es el lugar principal de acumulación de metales pesados, tanto de fuentes naturales como antropogénicas. Ante ello, se buscan técnicas de remediación in situ que son factibles, rentables y amigables con el medio ambiente; una de ellas, es la remedición mediante enmiendas orgánicas fácilmente disponibles que tienen la propiedad de inmovilizar el Cd mediante procesos de adsorción, complejación y precipitación (Clark et al., 2007; Bravo Realpe et al., 2014).

La aplicación de enmiendas orgánicas a los suelos agrícolas reduce la absorción de Cd por las plantas, mejora el suelo físicamente, además proporciona nutrientes esenciales para el suelo y las plantas. Sin embargo, algunas enmiendas pueden contener niveles elevados de $\mathrm{Cd}$ y, por lo tanto, su aplicación podría exacerbar los problemas de contaminación del suelo (Shaheen et al., 2014). Por lo cual, recomiendan determinar la concentración de Cd en las enmiendas orgánicas.

Las enmiendas orgánicas como el compost, residuos de estiércol, desechos orgánicos, biofertilizantes, residuos de cultivos, biosólidos y desechos sólidos municipales con bajo contenido de metal cumplen el papel fundamental en la remediación de suelos contaminados con metales pesados; por ende, son considerados fuentes de nutrientes y también como acondicionadores para la mejora de las propiedades físicas y la fertilidad de los suelos (tabla 3).

Las enmiendas orgánicas tiene la función de aumentar los sitios de unión, incrementar el potencial de hidrógeno del suelo, formar complejos estables y disminuir las biodisponibilidad del Cadmio (KabataPendias y Mukherjee, 2007).

\section{CONCLUSIONES}

De acuerdo al trabajo presentado, se pueden plantear las siguientes conclusiones principales:

1.- El uso de enmiendas orgánicas en suelos agrícolas contaminados con Cd es más realista, simple, factible, de rápida implementación, rentable y amigable con el medio ambiente.

2.- Las enmiendas orgánicas como el compost, vermicompost, biochar, residuos de cultivos, abonos de estiércol y desechos sólidos municipales con bajo contenido de $\mathrm{Cd}$ cumplen el papel fundamental en la mejora de la inmovilización del Cd en suelos agrícolas contaminados. 
3.-Las enmiendas orgánicas tienen la función de aumentar los sitios de unión, incrementar el potencial de hidrógeno del suelo, formar complejos estables y disminuir las biodisponibilidad del cadmio.

4.-En los últimos 13 años se ha realizado un considerable progreso en el uso de las enmiendas orgánicas y en el conocimiento de los procesos de adsorción, intercambio iónico, complejación y precipitación implicados en la inmovilización del cadmio.

5.- Los trabajos de remediación son complejos en la actualidad se tiene que realizar en base a un conjunto de técnicas (físicas, químicas y biológicas) para la obtención de mejores resultados, así como identificar la fuente o el origen de la contaminación para poder controlar el ingreso del contaminante en el suelo agrícola y luego optar por la remediación del suelo.

\section{REFERENCIAS}

Åkesson, A., Barregard, L. y otros cuatro autores, Non-Renal Effects and the Risk Assessment of Environmental Cadmium Exposure, https://doi.org/10.1289/ehp.1307110, Environmental Health Perspectives, 122(5), 431-438 (2014).

Ali, A., Guo, D. y otros cinco autores, Using Bamboo Biochar with Compost for the Stabilization and Phytotoxicity Reduction of Heavy Metals in Mine-contaminated Soils of China, https://doi.org/10.1038/s41598-017-03045-9, Scientific Reports, 7(1), 1-12 (2017).

Beesley, L., Moreno-Jiménez, E. y Gomez-Eyles, J.L., Effects of Biochar and Greenwaste Compost Amendments on Mobility, Bioavailability and Toxicity of Inorganic and Organic Contaminants in a Multi-element Polluted Soil, https://doi.org/10.1016/j.envpol.2010.02.003, Environmental Pollution, 158(6), 2282-2287 (2010).

Beesley, L. y Marmiroli, M., The Immobaliilisation and Retention of Soluble Arsenic, Cadmium and Zinc by Biochar, https://doi.org/10.1016/j.envpol.2010.10.016, Environmental Pollution, 159(2), 474-480 (2011).

Bhat, S.A., Singh, S. y otros cuatro autores, Bioremediation and Detoxification of Industrial Wastes by Earthworms: Vermicompost as Powerful Crop Nutrient in Sustainable Agriculture, https://doi.org/10.1016/..biortech.2018.01.003, Bioresource technology, 252, 172-179 (2018).

Bolan, N.S., Adriano, D.C., Duraisamy, P., y Mani, A., Immobilization and Phytoavailability of Cadmium in Variable Charge Soils. III. Effect of Biosolid Compost Addition, https://doi.org/10.1023/a:1026288021059, Plant and Soil, 256(1), 231-241 (2003).

Bravo, I.D.S.B, Arboleda, C.A.A, y Peinado, F.J M, Efecto de la Calidad de la Materia Orgánica asociada con el Uso y Manejo de Suelos en la Retención de Cadmio en Sistemas Altoandinos de Colombia, https://doi.org/10.15446/acag.v63n2.39569, Acta Agronómica, 63(2), 164-174 (2014).

Cha, J.S., Park, S.H. y otros cinco autores, Producción y Utilización de Biochar: una revisión, https://doi.org/10.1016/j.jiec.2016.06.002, Revista de Química Industrial y de Ingeniería, 40, 1-15 (2016).

Chaudri, A., McGrath, S. y otros siete autores, Cadmium Availability to Wheat Grain in Soils Treated with Sewage Sludge or Metal Salts, https://doi.org/10.1016/j.chemosphere.2006.09.068, Chemosphere, 66(8), 1415-1423 (2007).

Clark, G.J, Dodgshun, N., Sale, PWG., y Tang, C., Changes in Chemical and Biological Properties of a Sodic Clay Subsoil with Addition of Organic Amendments, https://doi.org/10.1016/ j.soilbio.2007.06.003, Soil Biology and Biochemistry, 39(11), 2806-2817 (2007).

Cloquet, C., Carignan, J. y otros tres autores, Tracing Source Pollution in Soil using Cadmium and Lead Isotopes. https://doi.org/10.1021/es052232+, Environmental Science \& Technology, 40(8), 2525-2530 (2006).

Cortes, L.E., Bravo, I.D.S., Martin., F.J. y Menjivar, J.C., Extracción Secuencial de Metales Pesados en dos Suelos Contaminados (Andisol y Vertisol) Enmendados con Ácidos Húmicos, https://doi.org/10.15446/acag.v65n3.44485, Acta Agronómica, 65 (3), 232-238 (2016).

Cruz-Paredes, C., Wallander, H., Kjøller, R., y Rousk, J., Using Community Trait-distributions to Assign Microbial Responses to $\mathrm{pH}$ Changes and Cd in Forest Soils Treated with Wood Ash, https://doi.org/10.1016/j.soilbio.2017.05.004, Soil Biology and Biochemistry, 112, 153-164 (2017).

De Melo, B.A.G., Motta, F.L., y Santana, M.H.A., Humic Acids: Structural Properties and Multiple Functionalities for Novel Technological Developments, https://doi.org/10.1016/j.msec.2015.12.001, Materials Science and Engineering C., 62, 967-974 (2016).

Geethamani, R., Soundara, B. y otros cinco autores, Affordable Compost Production Using Institutional Waste Using a Rapid Composting Method, https://doi.org/10.1016/j.matpr.2020.02.803, Materialstoday Proceedings, 105, 364-372 (2020).

Gramlich, A., Tandy, S. y otros cinco autores, Cadmium Uptake by Cocoa Trees in Agroforestry and Monoculture Systems under Conventional and Organic Management, https://doi.org/10.1016/j.scitotenv.2016.12.014, Science of The Total Environment, 580, 677-686 (2017).

Gramlich, A., Tandy, S. y otros cinco autores, Soil Cadmium Uptake by Cocoa in Honduras, https://doi.org/10.1016/j.scitotenv.2017.08.145, Science of The Total Environment, 612, 370-378 (2018). 
Guo, J., Dai, X., Xu, W., y Ma, M., Overexpressing GSH1 and AsPCS1 Simultaneously Increases the Tolerance and Accumulation of Cadmium and Arsenic in Arabidopsis Thaliana, https://doi.org/10.1016/j.chemosphere.2008.04.018, Chemosphere, 72(7), 1020-1026 (2008).

Guo, F., Ding, C. y otros tres autores, Stability of Immobilization Remediation of Several Amendments on Cadmium Contaminated Soils as Affected by Simulated Soil Acidification, https://doi.org/10.1016/j.ecoenv.2018.05.088, Ecotoxicology and Environmental Safety, 161, 164-172 (2018a).

Guo, F., Ding, C. y otros tres autores, Effects of Combined Amendments on Crop Yield and Cadmium Uptake in two Cadmium Contaminated Soils under Rice-wheat Rotation, https://doi.org/10.1016/j.ecoenv.2017.10.043, Ecotoxicology and Environmental Safety, 148, 303-310 (2018b).

Hait, S., y Tare, V., Vermistabilization of Primary Sewage Sludge, https://doi.org/10.1016/j.biortech.2010.10.031, Tecnología Bioresource, 102 (3), 2812-2820 (2011).

Hamid, Y., Tang, L. y otros cinco autores, Comparative Efficacy of Organic and Inorganic Amendments for Cadmium and Lead Immobilization in Contaminated Soil under Rice-wheat Cropping System, https://doi.org/10.1016/j.chemosphere.2018.09.113, Chemosphere, 214, 259-268 (2018).

Hao, X.Z., Zhou, D.M., Li, D.D., y Jiang, P., Cadmium and Zinc Accumulation of Ornamental Sunflower (Helianthus annuus L.) in Contaminated Soil with Different Amendments, https://doi.org/10.1016/S1002-0160(12)60048-4, Pedosphere, 22(5), 631-639 (2012).

He, S., He, Z. y otros tres autores, Soil Biogeochemistry, Plant Physiology, and Phytoremediation of CadmiumContaminated Soil, https://doi.org/10.1016/bs.agron.2015.06.005, Advances in Agronomy, 134, 135-225 (2015).

Houben, D., Pircar, J. y Sonnet, P., Heavy Metal Immobilization by Cost-effective Amendments in a Contaminated Soil: Effects on Metal Leaching and Phytoavailability, https://doi.org/10.1016/j.gexplo.2011.10.004, Journal of Geochemical Exploration, 123, 87-94 (2012).

Hueso-González, P., Muñoz-Rojas, M., y Martínez-Murillo, J.F., The Role of Organic Amendments in Drylands Restoration, https://doi.org/10.1016/j.coesh.2017.12.002, Current Opinion in Environmental Science \& Health, 5, 1-6 (2018).

Hu, Y., Cheng, H. y Tao, S., The Challenges and Solutions for Cadmium-contaminated Rice in China: A Critical Review, https://doi.org/10.1016/j.envint.2016.04.042, Environment International, 92-93, 515-532 (2016).

Irfan Sohail, M., Arif, M. y otros cinco autros, Organic Manures for Cadmium Tolerance and Remediation, https://doi.org/10.1016/b978-0-12-815794-7.00002-3, Cadmium Tolerance in Plants, 19-67 (2019).

Jiang, J., Xu, R., Jiang, T., y Li, Z., Immobilization of $\mathrm{Cu}$ (II), Pb (II) and Cd (II) by the Addition of Rice Straw derived Biochar to a Simulated Polluted Ultisol, https://doi.org/10.1016/j.jhazmat.2012.05.086, Journal of Hazardous Materials, 229-230, 145-150 (2012).

Juang, K.W., Ho, P.C. y Yu, C.H., Short-term Effects of Compost Amendment on the Fractionation of Cadmium in Soil and Cadmium Accumulation in Rice Plants, https://doi.org/10.1007/s11356-011-0684-0, Environmental Science and Pollution Research, 19(5), 1696-1708(2011).

Kabata-Pendias, A., y Mukherjee, A.B., Trace Elements from Soil to Human, 1ra edición, 550. Springer, Berlin, Alemania (2007).

Khan, M.A., Khan, S., Khan, A y Alam, M., Soil Contamination with Cadmium, Consequences and Remediation using Organic Amendments. https://doi.org/10.1016/j.scitotenv.2017.06.030, Science of The Total Environment, 601-602, 1591-1605 (2017).

Khan, M.A., Ding, X. y otros cuatro autores, The Influence of Various Organic Amendments on the Bioavailability and Plant Uptake of Cadmium present in Mine-degraded soil, https://doi.org/10.1016/j.scitotenv.2018.04.299, Science of The Total Environment, 636, 810-817(2018).

Kiran, Y.K., Barkat, A. y otros cinco autores, Cow Manure and Cow Manure-derived Biochar Application as a Soil Amendment for Reducing Cadmium Availability and Accumulation by Brassica chinensis L. in Acidic Red Soil, https://doi.org/10.1016/s2095-3119(16)61488-0, Journal of Integrative Agriculture, 16(3), 725-734 (2017).

Lewis, C., Lennon, A.M., Eudoxie, G., Umaharan, P., Genetic Variation in Bioaccumulation and Partitioning of Cadmium in Theobroma cacao L., https://doi.org/10.1016/j.scitotenv.2018.05.365, Science of The Total Environment, 640-641, 696-703 (2018).

Liao, Z., Wang, J. y otros cuatro autores, Immobilization of Cd in Landfill-Leachate-Contaminated Soil with Cow Manure Compost as Soil Conditioners: A laboratory Study. https://doi.org/10.1080/10962247.2016.1218976, Journal of the Air \& Waste Management Association, 66(12), 1276-1283 (2016).

Liu, L., Chen, H. y otros tres autores, Immobilization and Phytotoxicity of Cd in Contaminated Soil Amended with Chicken Manure Compost, https://doi.org/10.1016/j.jhazmat.2008.07.004, Journal of Hazardous Materials, 163(2-3), 563-567 (2009).

Liu, Y., Xiao, T. y otros cuatro autores, High Cadmium Concentration in Soil in the Three Gorges Region: Geogenic Source and Potential Bioavailability, https://doi.org/10.1016/j.apgeochem.2013.07.022, Applied Geochemistry, 37, 149156 (2013). 
Liu, K., Lv, J. y otros cuatro autores, Major Factors Influencing Cadmium Uptake from the Soil into Wheat Plants, https://doi.org/10.1016/j.ecoenv.2014.12.005, Ecotoxicology and Environmental Safety, 113, 207-213 (2015).

Liu, L., Li, W., Song, W y Guo, M., Remediation Techniques for Heavy Metal-Contaminated Soils: Principles and Applicability, https://doi.org/10.1016/j.scitotenv.2018.03.161, Science of The Total Environment, 633, $206-219$ (2018a).

Liu, L., Li, J. y otros cinco autores, Effects of Arbuscular Mycorrhizal Inoculation and Biochar Amendment on Maize Growth, Cadmium Uptake and Soil Cadmium Speciation in Cd-Contaminated soil, https://doi.org/10.1016/j.chemosphere.2017.12.025, Chemosphere, 194, 495-503 (2018b).

Liu, N., Jiang, Z. y otros cuatro autores, Mitigation of Rice Cadmium (Cd) Accumulation by Joint Application of Organic Amendments and Selenium (Se) in High-Cd-Contaminated Soils, https://doi.org/10.1016/j.chemosphere.2019. Chemosphere, 241, 125106 (2019).

R.M. N 0451-2018-MINAGRI, Aprueban Documento denominado "Lineamientos de Muestreo para la Determinación de Niveles de Cadmio en Suelos, Hojas, Granos y Productos derivados de Cacao", 4-6, Lima - Perú (2018).

Mohamed, I., Ahamadou, B. y otros cinco autores, Fractionation of Copper and Cadmium and their Binding with Soil Organic Matter in a Contaminated Soil Amended with Organic Materials, https://doi.org/10.1007/s11368-010-0199-1, Journal of Soils and Sediments, 10(6), 973-982 (2010).

Nieto-Velázquez, S., Pacheco-Hernández, L., Galán-Vidal, C.A. y Páez-Hernández, M.E., Estudio de las Interacciones Ácido Húmico-Metales Pesados y Determinación de sus Constantes de Estabilidad por Electroforesis Capilar, https://doi.org/10.4067/s0718-07642011000300007, Información Tecnológica, 22(3), 45-54 (2011).

Pan, L., Ma, J., Wang, X. y Hou, H., Heavy Metals in Soils from a Typical County in Shanxi Province, China: Levels, Sources and Spatial Distribution, https://doi.org/10.1016/j.chemosphere.2015.12.049, Chemosphere, 148, 248-254 (2016)

Pandey, D., Daverey, A., y Arunachalam, K., Biochar: Production, Properties and Emerging role as a Support for Enzyme Immobilization, https://doi.org/10.1016/j.jclepro.2020.120267, Journal of Cleaner Production, 255, 120267(2020).

Park, J.H., Lamb, D. y otros cuatro autores, Role of Organic Amendments on enhanced Bioremediation of Heavy Metal Contaminated Soils, https://doi.org/10.1016/j.jhazmat.2010.09.082, Journal of Hazardous Materials, 185(2-3), 549-574 (2011).

Rehman, M.Z., Zafar, M. y otros cinco autores, Residual Effects of Frequently Available Organic Amendments on Cadmium Bioavailability and Accumulation in Wheat, https://doi.org/10.1016/j.chemosphere.2019.125548, Chemosphere, 244, 125548 (2019).

Roberts, T.L., Cadmium and Phosphorous Fertilizers: The Issues and the Science, https://doi.org/10.1016/j.proeng.2014.09.012, Procedia Engineering, 83, 52-59 (2014).

Salmanzadeh, M., Hartland, A. y otros cinco autores, Isotope Tracing of Long-Term Cadmium Fluxes in an Agricultural Soil, doi: 10.1021/acs.est.7b00858, Environmental Science \& Technology, 51(13), 7369-7377 (2017).

Sarwar, N., Saifullah, S.S. y otros cuatro autores, Role of Mineral Nutrition in Minimizing Cadmium Accumulation by Plants, https://doi.org/10.1002/jsfa.3916, Journal of the Science of Food and Agriculture, 90, 925-937 (2010).

Sebastian, A. y Prasad, M.N.V., Cadmium Minimization in Rice. A review, https://doi.org/10.1007/s13593-013-0152-y, Agronomy for Sustainable Development, 34(1), 155-173 (2013).

Shaheen, S.M., Rinklebe, J. y Selim, M.H., Impact of Various Amendments on Immobilization and Phytoavailability of nickel and zinc in a contaminated floodplain soil, https://doi.org/10.1007/s13762-014-0713-x, International Journal of Environmental Science and Technology, 12(9), 2765-2776 (2014).

Shen, B., Wang, X. y otros cinco autores, The Optimum pH and Eh for Simultaneously Minimizing Bioavailable Cadmium and Arsenic Contents in Soils under the Organic Fertilizer Application, https://doi.org/10.1016/j.scitotenv.2019.135229, Science of The Total Environment, 711, 135229 (2019).

Singh, A. y Prasad, S.M., Effect of Agro-industrial Waste Amendment on Cd Uptake in Amaranthus caudatus grown under Contaminated soil: An Oxidative Biomarker Response, https://doi.org/10.1016/j.ecoenv.2013.09.005, Ecotoxicology and Environmental Safety, 100, 105-113 (2014).

Sun, Y., Xu, Y. y otros cuatro autores, Reliability and Stability of Immobilization Remediation of Cd Polluted Soils using Sepiolite under Pot and Field Trials, https://doi.org/10.1016/j.envpol.2015.10.054, Environmental Pollution, 208, 739-746 (2016).

Tajudin, S.A., Azmi, M.A. y Nabila, A.T., Stabilization/Solidification Remediation Method for Contaminated Soil: $A$ Review, https://doi.org/10.1088/1757-899x/136/1/012043, IOP Conference Series: Materials Science and Engineering, $136,1-6$ (2016).

Tang, W., Zhong, H. y otros cuatro autores, Inhibitory Effects of Rice Residues Amendment on Cd Phytoavailability: $A$ Matter of Cd-organic Matter Interactions?, https://doi.org/10.1016/j.chemosphere.2017.07.152, Chemosphere, 186, 227234 (2017). 
Wei, S., Li, Y. y otros cinco autores, Effect of Fertilizer Amendments on Phytoremediation of Cd-contaminated Soil by a Newly Discovered hyperaccumulator Solanum nigrum L., https://doi.org/10.1016/j.jhazmat.2009.11.023, Journal of Hazardous Materials, 176(1-3), 269-273 (2010).

Xie, Y., Xiao, K. y otros cuatro autores, Effects of Amendments on Heavy Metal Immobilization and Uptake by Rhizoma Chuanxiongon Copper and Cadmium Contaminated Soil, https://doi.org/10.1098/rsos.181138, Royal Society Open Science, 5(8), 1-12 (2018).

Yang, Y., Chen, J. y otros cinco autores, Can Liming reduce Cadmium (Cd) Accumulation in Rice (Oryza sativa) in Slightly Acidic Soils? A Contradictory Dynamic Equilibrium between Cd Uptake Capacity of Roots and Cd Immobilization in Soils, https://doi.org/10.1016/j.chemosphere.2017.11.061, Chemosphere, 193, 547-556 (2017).

Yousaf, B., Liu, G. y otros cinco autores, Investigating the Potential Influence of Biochar and Traditional Organic Amendments on the Bioavailability and Transfer of Cd in the Soil-plant System, https://doi.org/10.1007/s12665-0165285-2, Environmental Earth Sciences, 75(5), 1-10 (2016).

Yu, Y., Wan, Y., Camara, AY y Li, H., Effects of the Addition and Aging of Humic Acid-based Amendments on the Solubility of $\mathrm{Cd}$ in Soil Solution and its Accumulation in Rice, https://doi.org/10.1016/j.chemosphere.2018.01.002, Chemosphere, 196, 303-310 (2018). 
